\title{
A Nightingale-Based Model for Dementia Care and Its Relevance for Korean Nursing
}

\author{
Ann L. Whall, RN; PhD; FAAN \\ Professor, School of Nursing, University of Michigan, Ann Arbor \\ YunHee Shin, RN; PhD \\ Instructor, Department of Nursing, Yonsei University, Wonju-City, Kangwon-Do, Korea \\ Kathleen B. Colling, RN; PhD \\ Postdoctoral Fellow, School of Nursing, University of Michigan, Ann Arbor
}

\begin{abstract}
This article addresses the synchrony between a Western middle-range theory of care for persons with dementia and traditional Korean nursing care. The Western theory is called a need-driven, dementia-compromised behavior model and is heavily influenced by the assessment categories outlined in Nightingale's work. This model is presented as congruent with Nightingale's work and then viewed from the perspective of traditional Korean nursing. Several congruencies and a few incongruencies are found between these Western and Eastern views, and suggestions are made for greater consistency between these views.
\end{abstract}

The centrality of the concept of environment to nursing as a science is reflected in its inclusion across time as a major construct in various models of nursing (Johnson, 1980; Orlando, 1961; Roy, 1980), as coextensive with person (Levine, 1971; Rogers, 1970), or as the source of or an influence on the health or illness of the person (Flaskerud \& Halloran, 1980; Johnson, 1980; Neuman, 1980). Kim (1987) points out that the concept of environment is appropriate and necessary for nurse scientists to use to develop theories addressing influences on health and client recovery, the processes associated with environment interactions, and the nature of nursing practice within the environment itself. Nursing's tradition of viewing the environment as essential to its science was supported by early discussions of nursing's metaparadigm (Fawcett, 1978).

Authors' Note: Financial support for this project was granted to Kathleen B. Colling, recipient of a National Science Research Award postdoctoral fellowship in neurobehavior.

Nursing Science Quarterly, Vol. 12 No. 4, October 1999, 319-323

(C) 1999 Sage Publications, Inc.
Nightingale's emphasis on the environment as paramount to the achievement of health has been reflected in the evolution of middle-range theories focused on health in the Western tradition of nursing. Nightingale's work has also inspired nurse scientists schooled in both the Eastern and Western traditions to reflect on the synchrony of environmental focus to modern nursing. This article is an attempt to further explore a middle-range Western theory, that of the need-driven, dementiacompromised behavior (NDB) model (Algase et al., 1996), which seeks to improve health in dementia using an environmental focus. Nightingale's conceptualization of the environment (on which the NDB model is based) is also explored for its compatibility with Korean nursing.

\section{The Environment: Nightingale and the NDB Model}

Nightingale described two types of nursing, sick nursing and health nursing (Reed \& Zurakowski, 1996). Both sick nursing and health nursing were to "put the patient in the best condition for nature to act upon him" (Nightingale,
1859/1969). Nightingale viewed nursing and persons as having both the ability and the responsibility to alter the existing situations found noxious to health (Reed \& Zurakowski, 1996). This view was evident in her nursing discussions, which focus on actively changing the environment to improve conditions of both the individual and the community. Although her work may be viewed as simplistic and outdated, with limited applicability to modern nursing, which seeks a more holistic view of persons and their environment, Nightingale's discussions are relevant to the care of persons with compromised cognitive ability or dementia, for they guide the care of these persons within a restorative environment.

The NDB model focuses on dementia care based on the environmental view of Nightingale (Algase et al., 1996). The NDB model views the person with dementia as acting in the pursuit of a goal or personal needs. As in Nightingale's model, these persons are seen as affected greatly by their environment, which comprises the person,

Keywords: Korean nursing, Nightingale-based model 
the nurse, and other social and physical aspects of the environment.

Nightingale's nursing model has been described as focused almost exclusively on managing the environment so as to influence health (Reed \& Zurakowski, 1996). Nightingale's writings suggest that persons who are ill are vulnerable to a deleterious environment and, because they are often unable to alter their environment, nursing must take charge and make needed changes (Reed \& Zurakowski, 1996). The environment in these discussions of Nightingale clearly refers to physical and social elements external to the patient, those in particular that affect the health or healing process of the person. Physical elements such as noise, air quality, and temperature are all explicitly discussed by Nightingale. Social elements such as the actions of the nurse (rustling petticoats) and visitors (burdening the patient with worries) are also discussed.

The dementia care model of Algase et al. (1996) also addresses the physical and social environment of the demented through the concepts of background factors and proximal factors affecting the person with dementia. Background factors include the physical state, mental state, and other elements such as personal history of environmental preferences for the person with dementia. The proximal factors are current situational elements in the environment, which are both physical and social in nature. The physical aspect of environment is assessed (as within Nightingale's model) for obtrusive or disturbing sounds, problematic lighting and temperature, and many other features. The overall ambience of the environment is seen as particularly important; the ambience, in this sense, is the holistic influence of all physical and social aspects of the environment. Although the NDB model assesses multiple areas of/for the person with dementia, the final assessment retreats from a particulate view to assess the overall healthfulness of the ambient environment, which is integral to the person with dementia.

A healthful ambience is seen as one outcome of the interaction of back- ground and proximal factors in the NDB model. The ambience of the environment for persons with dementia is extremely important and must take into account their preferences and ability as persons embedded within their environment. If the person with dementia can no longer speak, but dislikes to interact with others, then being bathed in a nursing home in a noisy, crowded shower room presents a noxious ambient environment. In such a situation, a meaningful interaction with the person with dementia would be impossible to attain. If the caregiver also rushes through patient care and makes no pleasant remarks and/or has no eye contact or pleasant facial expression, a message is sent that the person with dementia is unimportant and that procedures, not people, are really what "count." If time is not made available for persons with dementia to maintain a sense of their selfworth (via a positive, ambient environment), their overall sense of self will suffer, leading perhaps to total withdrawal and even death. Thus, although an initial assessment of the NDB model focuses on specific aspects of the person and environment, the final appraisal concerns the holistic outcome of the environment-person interaction.

Reed and Zurakowski (1996) have suggested that Nightingale saw nursing practice as holistic in nature, encompassing spiritual, physical, emotional, social, or in essence, all aspects of a situation. Reed and Zurakowski (1996) emphasized that Nightingale's focus on specific elements within the environment is not "adding up the parts to apprehend the whole" (pp. 37-39) but, rather, is an attempt to capture the integrality of the environment-person interaction. One might also criticize the NDB model as "focusing on parts." However, the model is seen as a kaleidoscopic guide for consideration of the whole situation, including the ability and preferences of the person with dementia. The integration of these elements into a whole takes place within the conceptualization of the nurse, and this knowledge empowers the nurse to address the situation as a whole and take needed actions. Nightingale's model, as Reed points out, gave nurses the freedom to choose and to change the environment; Nightingale saw the interconnected wholeness of persons and all aspects of the environment.

The holistic view of Nightingale is also exemplified by her respect for the healing power of nature. Nightingale's goal of nursing, that of putting patients in the best position for nature to act upon them, demonstrates the importance of nature in her approach. When Nightingale prescribed a view from a window as facilitating the healing process, she predated the current findings within nursing and environmental psychology on the healing power of nature, arguably a holistic focus.

Recently, nurses and others have found evidence of the healing possibilities of a healthful ambient environment for persons with dementia (CohenMansfield \& Werner, 1998; Whall et al., 1997). Kaplan's (1983) model of the integrality of person-environment interactions describes a restorative environment as one that holds one's attention without struggle or effort, one that possesses a high level of coherence and intrigue, and one that promotes a high level of functioning. Natural environments have been found to facilitate a decrease in agitation in late-stage dementia, as well as other therapeutic effects, such as enhancing the ability of the demented person to speak and enjoy other aspects of the environment (Whall et al., 1997). It is ironic that a century after Nightingale's "laws of nature" were published, the use of nature to enhance the health of a special and very vulnerable population is becoming recognized.

Today, nurse educators and students in the United States are living in a global village, for students from multiple international communities attend all levels of nursing programs across the nation. This is a wonderful opportunity for the mutual exchange of nursing ideas and the comparative analysis of our bodies of knowledge. Because nursing within the United States is presented from a Western perspective, it is im- 
perative that international students examine such views for relevance to their own culture. Thus, the NDB model, with its reliance on Nightingale's work, is examined for relevance to Korean nursing. The issues addressed in this discussion are the relevance of the environmental view of the NDB model to Korean nursing and the acceptability of the NDB model's focus on natural elements to bring about health.

\section{Cultural Applicability of the NDB Model to Korean Nursing}

With an increase of Korean nurse scholars who have studied in Western countries, philosophies and beliefs relating to Western nursing have been introduced into Korea. As a result, traditional Korean nursing tended to be regarded as unscientific, since many Korean nurse scientists considered traditional nursing experiences and customs to be undeveloped and nonscientific folk remedies. However, because the person who provides care is Korean and the person who receives care is also Korean, the acceptance of Western nursing without reserve has sometimes resulted in conflicts or complications. Therefore, Korean nurse scientists are beginning to use traditional culture in the reconsideration of nursing care. Based on this reevaluation, Korean nurse scientists are making renewed efforts to develop a Korean body of nursing knowledge that is appropriate for Korean nurses, caregivers, and patients.

As previously discussed, Nightingale's model focused heavily on environment, especially on facilitating the patient's reparative processes through changes in the environment. Her nursing activity was directed toward the patient's environment as well as the patient. The NDB model also reflects the interaction of salient background and proximal factors found within the person with dementia as embedded in his or her immediate environment. Both Nightingale's and the NDB model's environmental focus are very similar to Korean traditional nursing care.

According to Eastern thought (including Korean thought), a human be- ing is born from nature, lives in nature, and returns to nature. Therefore, Korean people understand themselves within this context and live their lives accordingly. The Eastern view of life and nature (cosmos) finds harmony, not opposition, between a human being and nature (Bae, 1989). Eastern people have a nature-centered perspective in which they consider nature as a pattern or as a form of human life (Encyclopedia of Korean Traditional Culture, 1990; Grand Dictionary of Confucianism, 1990). Shin (1997), a Korean nurse scientist, explained that not only does the creation of the yin and yang symbolize the universe or the larger cosmos as a whole, it specifically represents a smaller cosmos consisting of a view of the body (combination of yin and yang), a view of life (circulation of yin and yang), and a view of health (harmony of yin and yang). Although a human being is considered a part of nature, the person is considered the most eminent microcosm among all creation. These views may assist Western nursing in the integration of partial knowledge of persons and their environment, achieving a holistic overall assessment.

The health of human beings depends on the harmony of yin and yang. If a person wants to be healthy, he or she should consider harmonizing with nature, which includes his or her surroundings as well as balanced human relationships in nature and society. Moreover, it is crucial for each person to reach hoshim, which means having a transcendental attitude about any kind of desire or keeping an open mind. Each person thus suppresses his or her excessive needs and attempts to remain aloof from the secular world. Hoshim can be viewed as a method of mind control to rise above the symptoms of disease in order to cope more effectively (Shin, 1997). Many Koreans view diseases as originating from their state of mind. Therefore, it is important to control thinking as the best way to preserve health.

The traditional Korean focus on the nurse caring for the person with a genuine desire to care and on helping the person to have a "comfortable mind" rather than on emphasizing the disease pro- cess is very important. In Korean nursing, the use of the natural environment and one's own self-healing power are also considered important methods of treatment, just as Nightingale focused on natural environments long ago (Kim, 1997). Throughout centuries, Koreans used their natural environment to cure diseases. Examples of this include recuperating within a beautiful natural environment with clean air or using medicinal plants from nature instead of chemical medicines. Traditional methods of treatment are sometimes disregarded, however, due to rapid development of modern medical therapies. Recently, knowledge of the value of natural therapies and the environment as methods in human self-healing are being widely reevaluated in Korea.

Another Korean nurse scientist, Lee (1997), emphasized that a human being and his or her environment have a mutual and interactive relationship rather than a separate one. Lee points to the balance of a human being and the environment as originating from an Eastern concept of moderation. Moderation means the state of no lack and no excess and also implies an unprejudiced state of mind. Kim, Han, and Shin (1994) reviewed the concept of environment as reflected in Korean traditional thoughts or philosophies of Shamanism, Buddhism, Confucianism, and Taoism. It was difficult to find the concept of environment as separate in these traditional patterns of thought. Instead, environment is represented in a view of nature and the universe. Even though the four traditional patterns of thought (Shamanism, Buddhism, Confucianism, and Taoism) differ somewhat in their views of nature, they all generally emphasize combination, harmony, and the antidichotomy (not being willing to divide into parts) of the human being and nature. Kim, Han, and Shin suggest that the concept of environment should be considered as an integrated concept in which the individual is not separate from but a part of his or her environment. Traditional Korean thought has been included in the Western unitarytransformative perspective (Newman, Sime, \& Corcoran-Perry, 1991), which 
addresses the view of the whole rather than focusing on combining parts. Holism is thus a construct long held in ancient Korean thought and tradition.

\section{Synchrony Between the NDB Model and Traditional Korean Nursing}

There is a synchrony between the concept of environment as addressed in Nightingale's model, Korean culture, and the NDB model for dementia care. Nightingale's perspective, although focused on the patient's environmental aspects such as ventilation, warmth, effluvia, noise, and light, emphasized the overall effect on the patient. The NDB model stresses a holistic view, which considers the patient's background and current situational factors that affect demented persons. The holistic view of Korean nursing would, however, focus more on the harmony of human beings and nature, such as having a "comfortable mind" or well-being of the mind, and the use of natural experiences.

Within the NDB model, the person with dementia is conceptualized as expressing a variety of need states or as communicating needs to achieve goals. Therefore, behaviors become meaningful because they can be potentially useful in directing nursing care. Within traditional Korean nursing, the human being is viewed as a smaller cosmos, which includes the body, life, and health. Because the person is considered the most eminent microcosm among all creation as a part of nature, nursing intervention can be directed toward self-healing and stimulation of hoshim.

Koreans traditionally value their surroundings and community highly, especially the family, by including the individual within family rather than just focusing on the individual. A health problem of a family member is regarded as a problem for the whole family, necessitating rearrangement of the environment. If someone in the family "has a disease," all members of the family address this member's care together and rearrange his or her environment for
NDBं Model

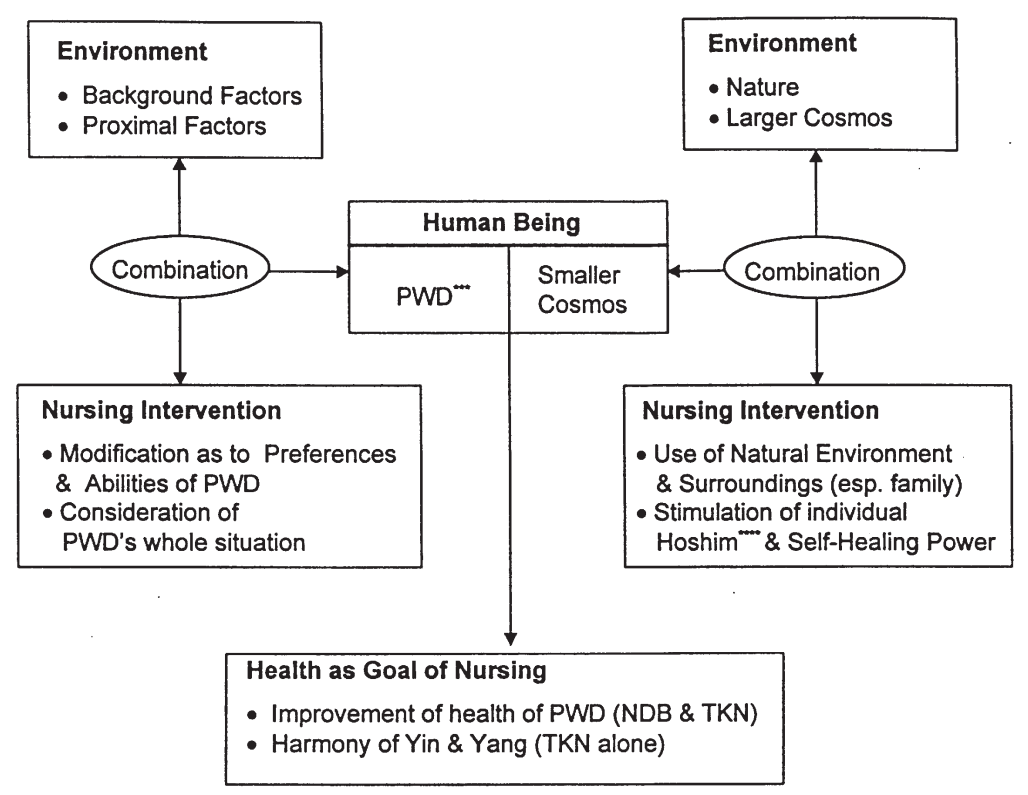

* NDB : Need-Driven Compromised-Behavior

* TKN : Traditional Korean Nursing

PWD : person with dementia

*** PWD : person with dementia

Hoshim : having transcendental attitude about any kind of desire or keeping an open mind

Figure 1. The Synchrony Between the Need-Driven, Dementia-Compromised Behavior Model and Traditional Korean Nursing

him or her to be comfortable rather than completely depending on a community facility. Likewise, if a father or mother has dementia, the children prefer cooperative care at home rather than in a long-term facility. Old pictures and possessions of their parents are used to stimulate past memories and provide a compatible environment. Parents might also be assisted to visit their hometown to return to familiar environments.

The NDB model assists the nurse in providing an environment compatible with preferences and abilities of the person with dementia, a perspective suitable for dementia care in Korea. The NDB model, although closely aligned with traditional Korean nursing, has a different focus on both environment and nursing. One of the differences between the two approaches has to do with the nature of the cosmos and with hoshim and self-healing as a component of nursing. The American culture may not address the person-environmental interrelationship in the same holistic manner of Korean culture; however, the goal of such care, that of providing a harmonious ambient environment that fosters health, is compatible with both cultures (see Figure 1).

In conclusion, to develop a body of nursing knowledge that is appropriate to their own culture, nurse scientists need to eclectically choose theories using integration strategies, autonomy, and flexibility (Lee, 1997). Western nursing undeniably possesses a body of knowledge that is well developed. However, if this knowledge is not in synchrony with a given culture, the application may not be useful. The examination of synchronous knowledge between existing cultures should foster a more useful body of knowledge. An understanding of how American nursing is alike and different from nursing found in other cultures allows for the consideration of new ways to view persons, environment, health, and nursing. Therefore, this article is one such attempt to foster such examination and suggests 
the usefulness of Korean viewpoints for American nursing. This view may enhance the delivery of care to patients and families in both the East and West.

\section{References}

Algase, D. L., Beck, C., Kolanowski, A., Whall, A., Berent, S., Richards, K., \& Beattie, E. (1996). Need-driven dementia-compromised behavior: An alternative view of disruptive behavior. American Journal of Alzheimer's Disease, 11(6), 10-19.

Bae, J. H. (1989). The philosophical development of Korean Confucianism. EeLee: Won Kwang University.

Cohen-Mansfield, J., \& Werner, P. (1998). The effects of an enhanced environment on nursing home residents who pace. The Gerontologist, 38, 199-208.

Encyclopedia of Korean traditional culture. (1990). Seoul: Academy of Korean Studies.

Fawcett, J. (1978). The "what" of theory development. In Theory development: What, why, how? (pp. 17-34). New York: National League for Nursing.

Flaskerud, J. H., \& Halloran, E. J. (1980). Areas of agreement in theory development. Advances in Nursing Science, 3(1), 1-7.

Grand Dictionary of Confucianism. (1990). Seoul: Bak Young.

Johnson, D. E. (1980). The behavioral sys- tem model for nursing. In J. Riehl \& C. Roy (Eds.), Conceptual models for nursing practice (2nd ed., pp. 207-215). New York: Appleton-Century-Crofts.

Kaplan, S. (1983). A model of personenvironment compatibility. Environment and Behavior, 15, 311-332.

Kim, H. S. (1987). Structuring the nursing knowledge system: A typology of four domains. Scholarly Inquiry for Nursing Practice: An International Journal, 1, 99-110.

Kim, M. S., Han, Y. R., \& Shin, Y. H. (1994). Review of environment in the Korean traditional thought. Korean Journal of Academy of Nursing, 24, 251-263.

Kim, S. J. (1997, December). Korean view of health. Paper presented at the meeting of Sigma Theta Tau International Honor Society of Nursing, Seoul.

Lee, Y. J. (1997, December). Korean view of nursing. Paper presented at the meeting of Sigma Theta Tau International Honor Society of Nursing, Seoul.

Levine, M. (1971). Holistic nursing. Nursing Clinics of North America, 6, 253263.

Neuman, B. (1980). The Betty Neuman health-care systems model: A total person approach to patient problems. In J. Riehl \& C. Roy (Eds.), Conceptual models for nursing practice (2nd ed., pp. 119-134). New York: AppletonCentury-Crofts.

Newman, M. A., Sime, A. M., \& CorcoranPerry, S. A. (1991). The focus of the dis- cipline of nursing. Advances in Nursing Science, 14(1), 1-6.

Nightingale, N. (1969). Notes on nursing: What it is, and what it is not (p. 133). New York: Dover. (Original work published 1859)

Orlando, I. (1961). The dynamic nursepatient relationship: Function, process and principles. New York: Putnam.

Reed, P., \& Zurakowski, T. (1996). Nightingale: Foundations of nursing. In J. Fitzpatrick \& A. Whall (Eds.), Conceptual models of nursing: Analysis and applications (3rd ed., pp. 27-54). Stamford, CT: Appleton \& Lange.

Rogers, M. E. (1970). An introduction to the theoretical basis of nursing. Philadelphia: F. A. Davis.

Roy, C. (1980). The Roy adaptation model. In J. Riehl \& C. Roy (Eds.), Conceptual models for nursing practice (2nd ed., pp. 179-192). New York: AppletonCentury-Crofts.

Shin, K. L. (1997). A study for the development of Korean nursing theory: Humanistic approach based on Shinhyung Naekyung pyun in Dongeuibogam. Korean Journal of Academy of Nursing, 27(1), 141-155.

Whall, A., Black, M., Groh, C., Yankou, D., Kupferschmid, B., \& Foster, N. (1997). The effect of natural environments upon agitation and aggression in late stage dementia patients. American Journal of Alzheimer's Disease, 11(6), 10-17. 